\title{
Research on Sports Information Technology Education Platform Based on ASP-NET Technology
}

\author{
Zhixin Zhang*
}

School of Physical Education, Langfang Teachers University, Langfang 065000, Hebei, China

\begin{abstract}
With the enhancement of information-based society, information technology has exceeded the single phase of computer technology training, developed and become information literacy education that satisfies demand of informationbased society. ASP.NET technology, enjoying features such as easiness to understand, and high efficiency of development, just adapts to the trend, and plays a crucial role in Web development, becoming one of the popular tools for development. Instruction of sports information technology course takes full advantage of Internet resources. It is quite necessary to conduct education by establishing website for sports instruction. Promote transformation of educational modes, reduce difficulty in organizing classes, increase efficiency and capacity of sports education and perfect educational assessment through development and practice of sports education platform.
\end{abstract}

Keywords: ASP-NET technology, education platform, sports information.

\section{SERVICE FRAMEWORK OF ASP-NET TECH- NOLOGY}

ASP.NET, which marks a server technology that combines various web elements as well as a uniform web development platform, provides various services required by a completed web application program. To a larger extent, grammar of ASP.NET is compatible with ASP. In addition, it also offers a new kind of program model and structure with the purpose of generating flexible application programs with higher security and stability. Compared with ASP, ASP.NET can provide better support to various browsers. It offers code compiling mechanism with clearer structures and easiness to write. It is quite easy to re-use and share these codes; it also improves configuration, flexibility, security and reliability. More importantly, ASRNET puts compiled language into application, thus improves flexibility. Besides, it also uses webform technology to conduct more intuitive development, and promotes reusing of components by utilizing object-oriented technology [1]. In addition, ASP.NET also includes page accidents, web control, buffer technology, server control and improvement on data binding. Core of NET platform is a series of new sets that is also named as .NET framework, which provides a platform that can develop WindowS-based programs and Web application programs in a rapid manner [2].

Net framework enjoys two primary components: common language runtime and .NET framework class lib, which offers fundamental technological support to the realization of .NET platform. Common language runtime, which is the executive engine of .NET platform, marks service established at the bottom layer of operating system [3]. NET framework includes a set of class lib that can be applied to any programming language, the purpose of which is making it easy for programmers to establish network-based application and service. Above the set are numerous models of application programs, which are capable of providing advanced components and service for network application and service development [4].

\section{STRUCTURAL FEATURES OF ASP.NET}

The two-layer application program, which means $\mathrm{C} / \mathrm{S}$ structure application program model in early phase, is primarily composed of customer end and database end. This program is relatively simple and clear and easy to develop. However, it does not enjoy perfect flexibility in the way that program written by developers can be operated at customer end by taking up a large number of system and network resources. Difficulty in development is increasing with the intensifying of application program service logistic as well as complexity. However, development of such system is relatively simple, and does not pose high requirements on technology of developers. Therefore, it enjoys certain market in application of internal LAN. The so-called three-layered system structure means adding a middle layer, which is also called as component layer between customer and database end (Fig. 1). The three-layered system referred to does not mean three layers in the physical aspect.

It means neither simply placing three machines nor enjoying B/S applications. Instead, it means three layers in the logical sense, in other words, placing the three layers in one machine, and work such as business rules, data access and validity identification etc. is placed and process in the middle layer by the application program. Under general circumstance, customer end does not interact with database; instead, it establishes connection with middle layer through COM/ DCOM communication, and conducts exchange with database through the middle layer (Fig. 2). Purpose of utilizing 


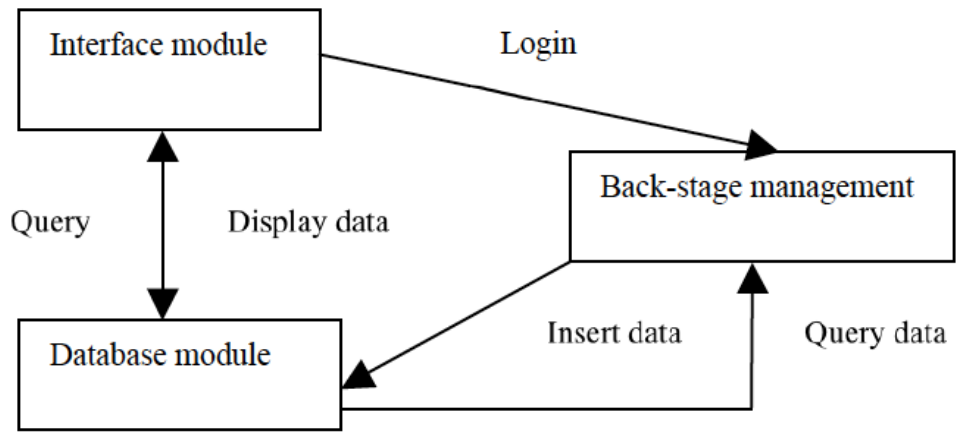

Fig. (1). The teacher information table.

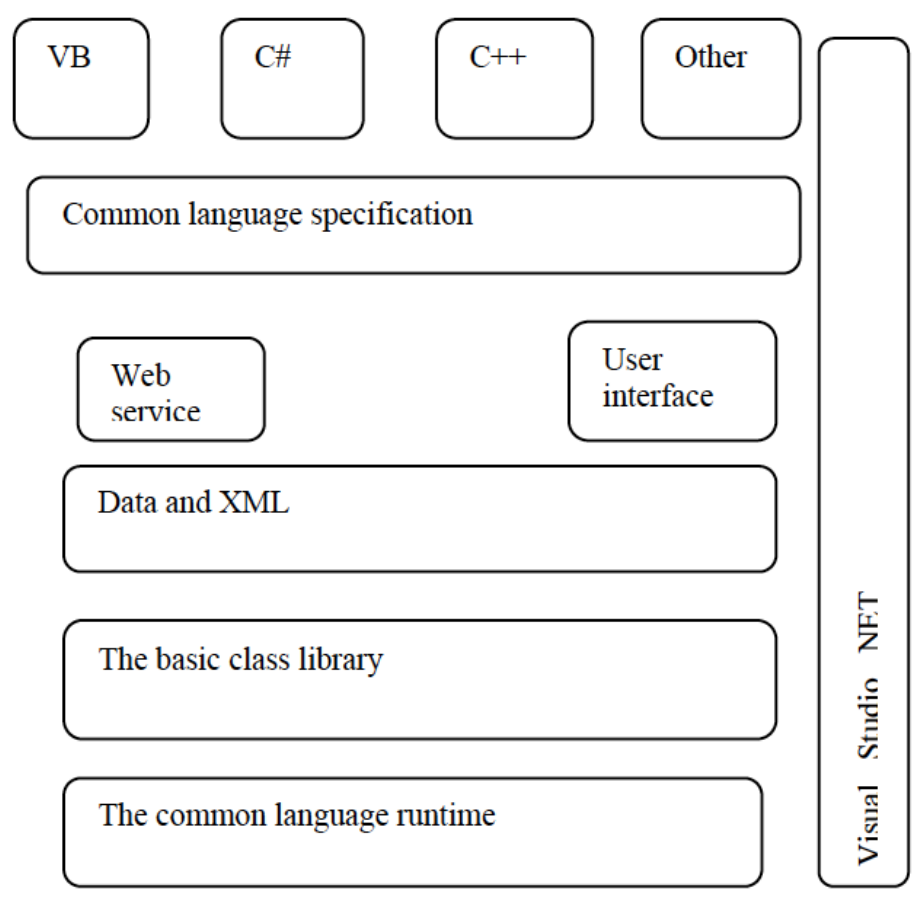

Fig. (2). Work information table.

the three-layered structure is to make program structure more clear, and division of labor more explicit, which is conducive for subsequent maintenance and update. The three-layered structure includes: USL, BBS and DAL.

\section{SYSTEM FEASIBILITY}

In the aspect of technology, the system conducts development and operation by putting into application the most extensively used ASP.NET + sQLserver2005, which marks the optimal choice from whether security, expansibility or stability. Meanwhile, the system, which can satisfy demands of preparation by teachers prior to classes, class instruction and independent study of students, can not only shorten distance between teachers and students by taking advantage of the Internet, but also be convenient for teachers to learn about students they teach, thus driving promotion of the whole. In addition, the system is easy to operate with strong instantaneity, for instance, on-line signing in, on-line practice of typing, and users can upload and unload required materials for studying after logging in. It perfects electronic platform of lesson planning for teachers and promotes their exchange and learning on teaching plans, completes electronic achieves of students' works, and generates scores of the process. Besides, it also perfects students' assessment platform (Including class performance, and work achievements), and promotes their interactive exchange and learning. It also completes records of class signing in and computer utilization, produces completed journeys in the computer room, perfects classroom testing platform and produces learning feedbacks immediately. The system, which enjoys easiness in operation, can be applied to different users, including both teachers and students.

\section{SYSTEM MODULE DESIGN}

Taking charge of interaction between system and users, interface module, which marks connector of the two, serves as the primary part to realize the function of interaction between people and computers. It can not only retrieve entry function in the database and searching content in it, but also log on management module at back stage and operate the database. Back-stage management module is the platform for administrators or teachers to update and maintain 


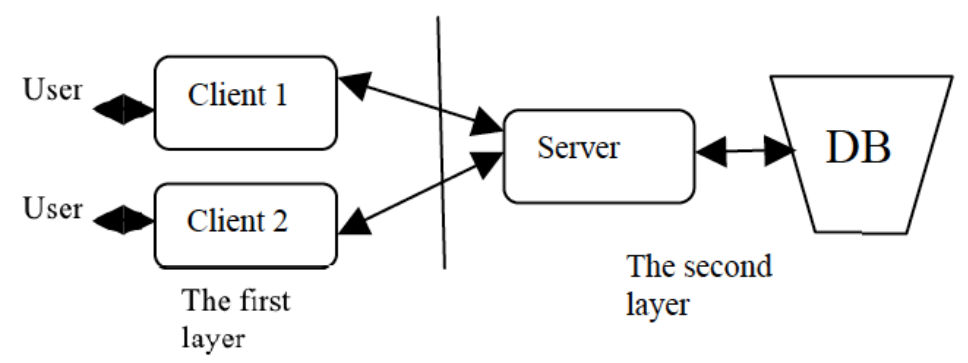

Fig. (3). C/S structure application model.

website content, through which other users can read the latest teaching resources and feedback information on the webpage (Fig. 3). Database module can define data structure, interface of the database, structure of database table, and automatically complete inputting, searching, modification of various information as well as management of system users and their rights (Fig. 4).

Software development environment

Windows2003Server

Operating system

SQLServer2005;

Database

Programming language

MierosoftVIS $\}$ alStudio.NET2008

Development tool

Software operation environment

Operating system:

WindowsXP+115/Windows200O/2003Server or others

Operating platform: Under B/S mode, IES.O/6.0 is required to be installed in the operating system.

\section{PROGRAMMING SOURCE CODE DESIGN}

Demonstrate signing in information of students on the page, and then record them in database.

DateTimetoda DateTime.Now;

intQyeatoday.Year:

intQmonth=today.Month;

intQday=today.Day:

intQgrade=Int32.Parse(Request.Cookies["Student Cookies"].

Values["Sgrade,,].Tostring()):

intQelass=Int32.Parse(Request.Cookies["Student Cookies"l.

Values["Selass"].Tostring()):

Learnsite.BLL.Signin59=newLearnsite.BLL.Signin():

DataListonline. Datasouree $=\mathrm{sg}$. OnlineToday $($ Qgrade, Qelass,Qyear,Qmonth,Qday);

DataListonline.DataBind();
Document of start.aspx.es on the page of teachers instructions:

Retrieve information in the database,and demonstrate students' signing in information on the page for teachers' instruction

intRgrade=Int32.Parse(DDLgrade.SeleetedValue);

intRelass=Int32.Parse(DDLelass.SeleetedValue);

DateTimedt=DateTime.Now;

intQyear=dt.Year;

intQmonth=dt.Month;

intQday=dt.Day:

LabelToday.Text-"+dt.ToLongDatestring();

Learnsite.BLL.Signin59=newLearnsite.BLL.Signin();

DLonline.Datasouree $=$ sg.StartsignClass $($ Rgrade, Rclass, Qyear,Qmonth,Qday):

DLonline.DataBind();

intdeount=DLonline. Items.Count;

Labelsigin.Text-"\&nbsp:"+deount.Tostring()+"\&nbsp;";

PrivatevoidShowNosigin()

intRgrade=Int32.Parse(DDLgrade. SelectedValue):

intRelass=Int32.Parse(DDLelass.SeleetedValue);

DateTimedt=DateTime.Now;

intQyear=dt.Year;

intQmonth=dt.Month;

intQday=dt.Day;

Learnsite.BLL.Signin59=newLeamsite.BLL.Signin():

DLnotline.Datasouree $=$ sg.StartNosignClass $($ Rgrade, Relass,Qyear,Qmonth,Qday);

DLnotline.DataBind();

Labelnosigin.Text-"\&nbsp:"+DLnotline.ltems.Count. Tostring ()+"

\&nbsP;":

sigllin.aspx.cs document on the interface of teacher signing in:

Information management of signing in by students. Teachers can conduct statistics on students' attendance in the whole semester. 
ProteetedvoidPage_Load(objeetsender,EventArgse)

LearnSite.Cornmon.CookieHelP.JudgeTeaeherCookies(): SetMai

Master.Page. Title=LearnSite.Common.CookieHelP.

if(!IsPostBaek)

if(Request.Cookies["T

GradeClass();

Showsignin();

Specifications are presented in the realization of the document: showmission.aspx.es.

Source codes are as follows:

if(Request.Querystring”Meid"]!=null)

\{stringWeid=Request.Querystring"Meid"].Tostring(): stringWmid=Request.Querystring"Mid"\} .Tostring():

if(LearnSite.Common.WordProeess.IsNum(Weid)\&\&

LearnSite.C0mmon.WordProeess.IsNum(Wmid))

Learnsite,BLL.Worksws $=$ newLean 1 SiteBLL.Works();

StringSnumDone $=$ Ws.IPWorkDonesnum(Int32.Parse (Sgrade),Int32.Parse(Selass),Int32.Parse(Weid),Int32.

Parse(Wmid),WIP):

if(SnumDone=="')

if(LabelMletyPe.Text!=’html"

Labelmsg.Text=DateTime.Now.ToshortDatestring();

if(Snum!=SnumDone)

if(LearnSite.Common.XmlHelP.GetWorkIPLimit())

AttaehFile.Enabled=false;

Button of handing in will be grey and expired if the setting of work handing in is IP limitation.

Labelmsg. Text $=$ \}

Labelmsg. Text $=$ \}

Task work has been handed in at the IP address.

else

if(Session[Snum]!=null)

AttaehFile.Enabled=false;

BtnuPload.Enabled=false;

You have participated in the vote.

No re-handing in is allowed.

CISe

The work can be modified

stringWid=ws.WorkDone(Snum,Int32.Parse(Wcid),

Returning to null characters means the record does not exist.

if(Wid"')

Button of handing in will be grey and expired if the work has been marked
if(ws.IsCheeked(Int32.Parse(Wid)))

The work has been marked

BtnuPload.Enabled=false;

AttachFile.Enabled=false;

CheekBoxCan.Enabled=false;

else

if(CheekBoxMuPload.Cheeked)

\}

else

\{

Panelworks. Visible $=$ false;

Time-out and expired web page

stringPostfilename $=$ this.AttaehFIle.FIleName;

intWlength $=$ Convert.Tolnt32(this.AttaehFile.Content

Length);

if(Postfilename!="' \&\&Postfilenaxne!null)

if(LeamSite.Common.WorkUPload.CheekTyPe(Postfilen ame,

Whether the type is right or not.

Learnsite.BLL.Worksws=newLearnsite.BLL.Works(); stringWid=ws.WorkDone(Wnum,Int32.Parse(Weid)

Returning to null characters means the record does not exist.

if(Wid!="'”)

Re-hand in modified work

stringMysavePath=

Obtain work saving path (Automatically establish in case of no existence)

stringRndTime $=$

(DateTime.Parse(LoginTime)).Minute.Tostring(): stringNewFileName $=$ Server.UrlDeeode $($ Sname $)+$ Wcid + Wmid+_,+RndTime+iPlast+.+MfiletyPe:

stringWurl=MysavePath+"/"'+NewFileName;

stringsaveFilename=Server.MaPPath(Wurl):

try $\{$ this.AttaehFile.MoveTo(saveFilename,Bret-

tle.Web.NeatUPload.MoveToOPt

ws.UPdateworkUP(Int32.Parse(Wid),Wurl,NewFileName, Wlength,

Curriculum activity, update,Succeed in re-handing in work

Labellnsg.Text-ch;

LearnSite.Common.WordProcess.Alert(eh,this.Page):

eateh(ExeePtionex)

\{

thLrowex; 


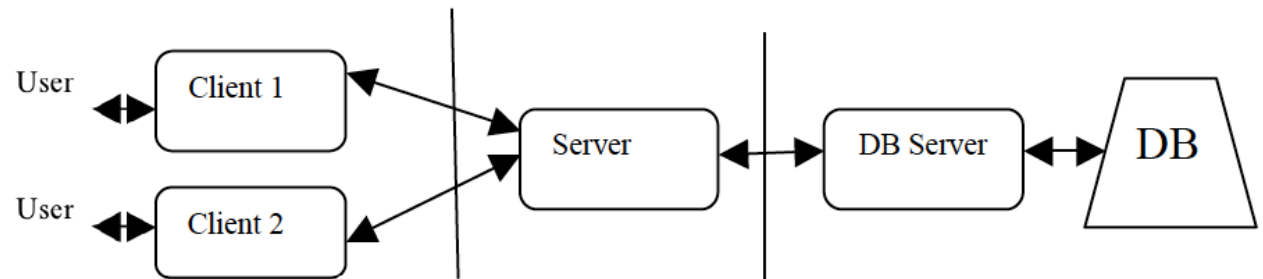

The first layer

The second layer

The third layer

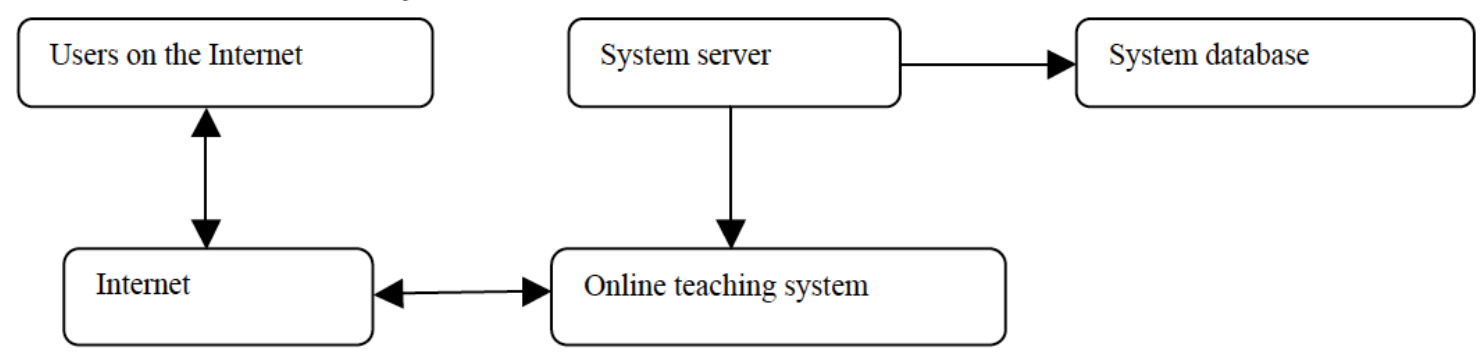

Fig. (4). Three layer system structure.

\section{CONCLUSION}

With the enhancement of information-based society, information technology has exceeded the single phase of computer technology training, developed and become information literacy education that satisfies demand of informationbased society. ASP.NET technology, enjoying features such as easiness to understand, and high efficiency of development, just adapts to the trend, and plays a crucial role in Web development, becoming one of the popular tools for development. Instruction of sports information technology course takes full advantage of Internet resources. It is quite necessary to conduct education by establishing website for sports instruction. Promote transformation of educational modes, reduce difficulty in organizing classes, increase efficiency and capacity of sports education and perfect educational assessment through development and practice of sports education platform.

\section{CONFLICT OF INTEREST}

The author confirms that this article content has no conflict of interest.

\section{ACKNOWLEDGEMENTS}

Declared none.

\section{REFERENCES}

[1] X. Qiang, “ASP.NET and sport information service," Journal of Chengdu Sports Institute, vol. 29, pp. 34-38, 2010.

[2] Q. Keng, G. Jingpin, and H. Dongtian, "Study on modern sport information industry," Journal of Beijing Sport University, vol. 41, pp. 3-9, 2011.

[3] D. Guo, and P. Huang, "Development model of ASP.NET," Journal of Heibei University, vol. 41, pp. 123-133, 2011.

[4] G. Feige, The ASP.NET Information System, Nanchang: Nanchang University Publishing, 2011, pp. 22-76.

Received: June 10, 2015

Revised: July 29, 2015

Accepted: August 15,2015

(C) Zhixin Zhang; Licensee Bentham Open.

This is an open access article licensed under the terms of the (https://creativecommons.org/licenses/by/4.0/legalcode), which permits unrestricted, noncommercial use, distribution and reproduction in any medium, provided the work is properly cited. 